\title{
Galaxy Pairs and Triplets at Medium Redshifts from the Sloan Digital Sky Survey (SDSS)
}

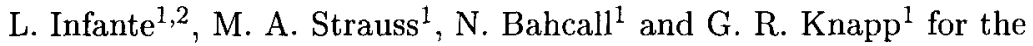 \\ SDSS Collaboration
}

\begin{abstract}
We have identified close pairs and triplets of galaxies in SDSS commissioning imaging data. The data set is from one of the SDSS equatorial scans covering an area close to 100 square degrees. We have estimated the angular correlation function of these galaxy systems and show that they appear to be appreciably more strongly clustered than are single galaxies.
\end{abstract}

\section{Introduction}

Numerous studies have been carried out to understand the clustering properties of galaxies and of clusters of galaxies. A central issue is the existence of a common physical mechanism to explain their clustering properties. Pairs and triplets of galaxies fill in the gap in the hierarchy between individual galaxies and clusters of galaxies.

The aim of this project is to study the statistical relations between compact isolated galaxy systems and the field galaxy population. In this paper, we examine the angular correlation function of compact pairs and triplets of galaxies. (See Infante et al. 1999 for a detailed report.)

The SDSS commissioning data provide a photometrically reliable catalog of galaxies on a large field to $r^{*} \approx 20.5$. Compact poor groups have been detected and classified over an area of $\sim 96.5 \mathrm{deg}^{2}$.

\section{SDSS Data}

The Sloan Digital Sky Survey is a photometric and spectroscopic survey of about one quarter of the sky, above Galactic latitude about $30^{\circ}$. It is described by Gunn \& Weinberg (1995), Strauss et al. (1999) and, in detail in the SDSS "Black Book" (http://www astro.princeton.edu/BBOOK/). The photometric data are taken with a dedicated $2.5 \mathrm{~m}$ altitude-azimuth telescope at Apache Point, New Mexico, with a $2.5^{\circ}$ wide distortion-free field (Siegmund et al. 1999) and an imaging camera consisting of a mosaic of 30 imaging $2048 \times 2048$ SITe CCDs with $0.4^{\prime \prime}$

\footnotetext{
${ }^{1}$ Department of Astrophysical Sciences, Princeton University, Peyton Hall, Princeton, NJ 085441001, U.S.A.

${ }^{2}$ Departamento de Astronomía y Astrofísica, P. Universidad Católica de Chile, Casilla 306, Santiago 22, Chile
} 
pixels (Gunn et al. 1998). The CCDs are arranged in six columns of five CCDs each, each column enclosed in a single dewar and observing the sky through five broad-band filters $\left(u^{\prime}, g^{\prime}, r^{\prime}, i^{\prime}\right.$ and $\left.z^{\prime}\right)$ which cover the optical spectral range from the atmospheric cutoff in the ultraviolet to the silicon sensitivity cutoff in the red.

The data reach to roughly $r^{*}=22.5$ in Equatorial scans in the Southern Galactic Cap which we use in this paper ${ }^{1}$. Star-galaxy separation is done to $r^{*} \approx 21$ by fitting a local PSF to each $10^{\prime} \times 13^{\prime}$ frame, and drawing a line in the magnitude/central surface brightness plot. Petrosian magnitudes are used in this paper.

128,937 galaxies were found in the range $17 \leq r^{*} \leq 20$, over an area of $96.5185 \mathrm{deg}^{2}$. The seeing was $1.2^{\prime \prime}-2.0^{\prime \prime}$. The photometric zero points were calibrated by observing secondary standards in the survey area (Smith et al. 1998, Tucker et al. 1998) using the $24^{\prime \prime}$ APO and the US Naval Observatory's $40^{\prime \prime}$ telescopes.

We select projected galaxy pairs and triples so that at $r^{*} \approx 20,>50 \%$ of the systems would be physical, and not projected (i.e., $w(\theta) \geq 1$ ). For a mean sample redshift of $z=0.24$, computed from the CNOC2 luminosity functions (Lin et al. 1999), these conditions are satisfied by group radius $R_{G} \simeq 37.3 h^{-1}$ kpc, which corresponds to $\sim 15^{\prime \prime}$.

We selected only pairs and triples with no neighbors within a distance three times the group radius to the center of the group. Our group algorithm selects pairs and groups of galaxies within $17<r^{*}<20$ and $2^{\prime \prime}<\theta<15^{\prime \prime}$.

Out of the original 128,937 galaxies, we found 6,769 pairs of galaxies $(10.5 \%$ of the galaxies are in pairs) and 550 triplets of galaxies (1.28\% of the galaxies are in triplets).

\section{Correlation Function}

To estimate the galaxy angular correlation function, $w(\theta)$ we used the estimator derived by Landy \& Szalay (1993):

$$
\omega(\theta)=\frac{N_{d d}-2 N_{d r}+N_{r r}}{N_{r r_{1}}}
$$

where $N_{d d}$ is the number of data pairs in a given range of separations summed over all SDSS fields, $N_{d r}$ is the number of random pairs using galaxies as centers, $N_{r r}$ is the number of random pairs, and $N_{r r_{1}}$ is the same as $N_{r r}$ but an independent set of random counts. Pair counts were done in each column, or scanline, of SDSS data separately.

Considering that the pairs were selected on the basis of $w(\theta)>1$, it is entirely consistent that half of the pairs are physical systems (Carlberg, Pritchet \& Infante 1994), and from our Monte Carlo simulations (Infante et al. 1999) we find that $\sim 1 \%$ of the galaxies are in real triple systems at $\langle z\rangle \sim 0.24$.

\footnotetext{
${ }^{1}$ Because the photometric calibration is not finalized, we refer to observed photometry with asterisks, not the primes of the final system.
} 
To obtain the correlation length $r_{o}$ we compute standard models by inverting Limber's equation. We assume a power-law spatial correlation function, $\xi(r, z)=$ $\left(\frac{r}{r_{o}}\right)^{-\gamma}(1+z)^{-(3+\epsilon)}$ where $r$ is the proper distance, $r_{o}$ is the proper correlation length, and $\epsilon$ is the clustering evolution index. This inversion depends on the redshift distribution $d N / d z$. To compute $d N / d z$ we used CNOC2 luminosity functions published in Lin et al. (1999), after proper band transformation.

\section{Results}

The results are shown in Fig. 1 and Table 1. The galaxy angular correlation function follows a power law, $\omega=A_{\omega} \theta^{(1-\gamma)}$, with $\gamma=1.8$ and an amplitude consistent with previous estimates (Infante \& Pritchet, 1995). Moreover, it does not show any breaks at large angular scales. The error bars in the galaxy $w(\theta)$ are smaller than the symbols.

$w(\theta)$ for galaxy pairs and triplets shows the same power law behavior as for galaxies but with an amplitude that increases as a function of the number of group members. Contamination by random objects decreases the amplitude, leaving the shape of $w(\theta)$ unaltered. According to our simulations, if $\sim 30 \%$ of the pairs are not physical, then $r_{0} \approx 11 h^{-1} \mathrm{Mpc}$, and if $\sim 20 \%$ of the triplets are chance superpositions, then $r_{o} \approx 18 h^{-1} \mathrm{Mpc}$.

We have also estimated that the fraction of galaxies in close triple systems, at a mean redshift of $0.3\left(19<r^{*}<21\right)$, is $1.9 \%$. If account is taken of possible triplets detected due to superposition, the fraction of galaxies in physical triplets at $z=0.3$ is $0.9 \%$.

Table 1. SDSS Run 94: Catalog $17 \leq r^{*} \leq 20$

\begin{tabular}{lccc} 
Property & Galaxies & Pairs & Triplets \\
\hline$\langle z\rangle$ & $0.24 \pm 0.10$ & $0.24 \pm 0.10$ & $0.24 \pm 0.10$ \\
$D\left[{ }^{\prime \prime}\right]$ & 15.0 & 15.0 & 15.0 \\
Volume $\left[h^{-3} \mathrm{Mpc}^{3}\right]$ & $7,700,000$ & $7,700,000$ & $7,700,000$ \\
$\left\langle r^{*}\right\rangle[$ SDSS band $]$ & 19.25 & 19.25 & 19.25 \\
Number $(\%)$ & $128,937(100)$ & $6,769(10.50)$ & $550(1.28)$ \\
$A_{\omega},(\gamma)$ & $5.02(1.77)$ & $15.64(1.79)$ & $34.89(1.75)$ \\
$A_{\omega},(\gamma=1.8)$ & $5.79(1.80)$ & $16.69(1.80)$ & $48.58(1.80)$ \\
$r_{o}\left[h^{-1} \mathrm{Mpc}\right]$ & 4.5 & 8.0 & $14.5 \pm 2.0$ \\
\hline
\end{tabular}

${ }^{a} h=H_{0} / 100, q_{0}=0.1, D_{\max }=37.3 \mathrm{kpc}$, Area $_{\text {Total }}=96.5 \mathrm{deg}^{2}$

We conclude that pairs and triplets of galaxies appear to be appreciably more strongly clustered than are single galaxies. These results are based on early SDSS commissioning data; as the data increases in both quality and quantity, these results can be investigated much more thoroughly.

Acknowledgments. The Sloan Digital Sky Survey (SDSS) is a joint project of The University of Chicago, Fermilab, the Institute for Advanced Study, the 


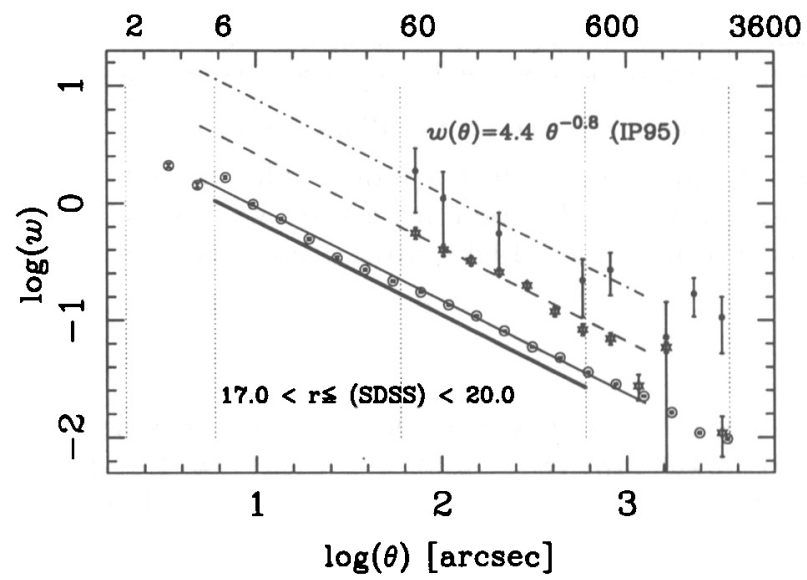

Figure 1. Angular Correlation Function of galaxies, pairs and triplets for $17 \leq r^{*} \leq 20$. The dark solid line is the IP95 (Infante \& Pritchet 1995) $w(\theta)$ result with $\delta=-0.8$. The thin solid line is the model for galaxies with $\left(r_{o}=4.5 h^{-1} \mathrm{Mpc}\right)$. The dashed line is a model for pairs with $\left(r_{o}=8.0 h^{-1} \mathrm{Mpc}\right)$. The dashed dotted line is a model for triplets with a large correlation length $\left(r_{o}=14.5 h^{-1} \mathrm{Mpc}\right)$. All models are for $\epsilon=0.8$.

Japan Participation Group, The Johns Hopkins University, the Max-PlanckInstitute for Astronomy, Princeton University, the United States Naval Observatory, and the University of Washington. Apache Point Observatory, site of the SDSS, is operated by the Astrophysical Research Consortium. Funding for the project has been provided by the Alfred P. Sloan Foundation, the SDSS member institutions, the National Aeronautics and Space Administration, the National Science Foundation, the U.S. Department of Energy, and the Ministry of Education of Japan. We are grateful to Fundación Andes for providing part of the financial support, to Princeton University for its hospitality and to FONDECYT project \#8970009. The IAU kindly provided a travel grant to the meeting.

\section{References}

Carlberg, R., Pritchet, C., \& Infante, L. 1994, ApJ, 435, 540

Fukugita, M., Ichikawa, T., Gunn, J. E., Doi, M., Shimasaku, K., \& Schneider, D.P. $1996, \mathrm{AJ}, 111,1748$

Gunn, J. E., et al. , 1998, AJ, 116, 3040

Gunn, J. E., \& Weinberg, D., 1995, in "Wide Field Spectroscopy and the Distant Universe", ed. S. Maddox \& J. Aragón-Salamanca, World Scientific, Singapore, 3

Infante, L. et al. , 1999, in preparation

Infante, L. \& Pritchet, C. 1995, ApJ, 439, 565

Landy, S. D. \& Szalay, A. S., 1993, ApJ, 412, 64 
Lin H., et al. 1999, AJ, in press, (astro-ph 9902249)

Siegmund, W. et al., 1999, in preparation

Smith, J. A. et al., 1998, BAAS, 30, 1244

Strauss, M. A., et al. 1999, in "Astrophysics with Infrared Surveys: A Prelude to SIRTF", ed. M. Bicay, ASP Conf. Proceedings (in press)

Tucker, D. L. et al., 1998, BAAS, 30, 1245 